\title{
ESTUDIO COMPARATIVO DEL RENDIMIENTO ENTRE NIÑOS REPETIDORES Y NO REPETIDORES
}

\author{
Alar Urruticoechea \\ Universidad Católica del Uruguay (UCU), Uruguay. \\ alar.urruticoechea@ucu.edu.uy \\ Elena Vernazza \\ Instituto de Estadística, Facultad de Ciencias Económicas y de Administración, \\ Universidad de la República (UdelaR), Uruguay. \\ Diana del Callejo Canal \\ Instituto de Investigación de Estudios Superiores, \\ Económicos y Sociales de la Universidad Veracruzana (IIESES-UV) \\ Margarita Canal Martínez \\ Instituto de Investigación de Estudios Superiores, \\ Económicos y Sociales de la Universidad Veracruzana (IIESES-UV) \\ Ramón Álvarez Vaz \\ Instituto de Estadística, Facultad de Ciencias Económicas y de Administración, \\ Universidad de la República (UdelaR), Uruguay.
}

Recepción Artículo: 1 marzo 2020

Admisión Evaluación: 4 marzo 2020

Informe Evaluador 1: 8 abril 2020

Informe Evaluador 2: 22 marzo 2020

Aprobación Publicación: 20 abril 2020

\section{RESUMEN}

Introducción: Hoy en día el Sistema Educativo español propone la repetición como herramienta niveladora entre el rendimiento real del escolar y el nivel académico al que concurre. Si bien se ha demostrado que las consecuencias negativas de la repetición a corto, medio y largo plazo son mayores que los beneficios, la posibilidad de utilizar este mecanismo ha convertido a España en el país de la OCDE con mayor tasa de repetidores. Tomando este dato como punto de partida surge la duda de si realmente la repetición está cumpliendo con el objetivo para el cual fue creada. Objetivo de investigación: Comparar los niveles de rendimiento entre los estudiantes repetidores y los no repetidores. Método: Para lograr este objetivo se analizaron 25245 respuestas de alumnos de cuarto de Educación Primaria a la prueba "Evaluación General de Diagnóstico" de 2009. Las estrategias metodológicas utilizadas fueron, por un lado, el análisis de diferencia de medias, en particular, mediante la prueba Welch y por otro, la metodología HJ-Biplot. Resultados: Del total de la muestra el $6.83 \%$ son repetidores. Todas las competencias a estudio (Comunicación lingüística, Matemática, Interacción con el mundo físico y Social y ciudadana) se asocian positivamente entre ellas, donde la correlación más fuerte se da entre Mundo físico y Lingüística (.68) y entre Mundo físico y Social- 
Ciudadana (.68), y la más débil entre Social-Ciudadana y Lingüística (.57). Los alumnos no repetidores obtienen puntuaciones medias más altas en todas las competencias y estas diferencias son significativas obteniendo un p. valor <.001 en la prueba de Welch. Conclusión: La repetición, en la mayoría de los casos, no sirve para mejorar el rendimiento. Discusión: Entendiendo que la repetición genera desigualdad de los niños en la sociedad y que no es una herramienta útil, es necesario realizar otro tipo de intervenciones para mejorar el rendimiento de los alumnos que son propensos susceptibles a repetir.

Palabras clave: repetición; rendimiento académico; inclusión; HJ-Biplot; prueba de Welch

\section{ABSTRACT \\ Comparative study of performance between grade repetition children and non-grade repetition children}

Introduction: The Spanish Educational System proposes repetition as a leveling mechanism of the real performance of the scholar and their academic level. Although the negative consequences of repetition in the short, medium and long term have been shown to outweigh the benefits, the possibility of using this mechanism has made it the OECD country with the highest rate of repeaters. With this as a starting point, the question is if the repetition is fulfilling the aim for which it was created. Research objective: Grade repetition children and non-grade repetition children performance comparation. Method: To achieve this aim, were analyzed 25,245 responses of fourth grade students of Primary Education to the test "Evaluación General de Diagnóstico" of 2009. The methodological strategy used is mean difference analysis, using the Welch test and the HJ-Biplot. Results: $6.83 \%$ of the sample are repeaters. All study competencies (Linguistic communication, Mathematics, Interaction with the physical and Social world and citizens) correlate positively, the strongest correlation is between the physical and Social world and linguistics (.68) and between the physical and Social world and citizen (.68), and the weakest correlation between citizen and linguistics (.57). Non-grade repetition students obtain higher average scores in all the competences and these differences are significant ( $p$-value <.001) in the Welch test. Conclusion: in most cases, Repetition does not serve to improve performance. Discussion: repetition generates inequality for children in society and that it is not a useful mechanism, it is necessary to carry out other types of interventions to improve the performance of students who are susceptible to repeat.

Keywords: repetition, academic performance, inclusion, HJ-Biplot, Welch test

\section{INTRODUCCIÓN}

La repetición se ha convertido en un mecanismo para equiparar el rendimiento cognitivo de los escolares a las exigencias de cada nivel educativo, por lo cual, en España el escolar que no logra los objetivos y no alcanza el grado de adquisición de las competencias correspondientes es susceptible a repetir curso (Ley Orgánica 2/2006, de 3 de mayo). La posibilidad de utilizar este mecanismo ha convertido a España en el país de la OCDE con mayor tasa de repetidores (Cabrera, 2019).

Partiendo de investigaciones previas, en niveles socioeconómicos bajos, se puede asegurar que la repetición, inicialmente puede conducir una mejora en el rendimiento (Magnuson et al., 2004), posteriormente puede conllevar repercusiones negativas a corto, mediano y largo plazo (GonzálezBetancor \& López-Puig, 2016). A corto plazo, existe mayor probabilidad de abandono educativo entre los escolares repetidores que entre los que son promocionados de manera continua (Hughes et al., 2017). A medio plazo, podrían existir mejoras en la atención de los escolares, pero no en los 
resultados académicos (Mantzicopoulos \& Morrison, 1992). Finalmente, a largo plazo, los beneficios de la repetición son menores que los perjuicios provocados, especialmente a nivel emocional (Hemelt \& Rosen, 2016), relacionándose con niveles más bajos en formación, salario y capacidad de ahorro (Peña, 2017).

La mayoría de las investigaciones se centran en las repercusiones a largo plazo de la repetición, pocas se centran en verificar si se cumple el objetivo para el cual se propone esta herramienta. España, concretamente, a partir de la utilización de este mecanismo se ha convertido en el país de la OCDE con mayor tasa de repetidores (Cabrera, 2019). Por lo que esta investigación se centra en las repercusiones que tiene la repetición a corto plazo, especialmente en el rendimiento de los escolares. Se entiende por rendimiento el nivel de conocimientos, habilidades y destrezas alcanzados por el escolar (Barbosa, 1975). Este puede ser evaluado mediante pruebas académicas puntuales (exámenes de aula), pruebas estandarizadas que miden el logro académico internacionalmente (pruebas PISA) y pruebas estandarizadas realizadas a nivel nacional (Evaluación General de Diagnóstico (EGD), realizada en España (Instituto de Evaluación (España), 2010)). Esta última prueba es la que se utilizará en esta investigación.

\section{Objetivos de la investigación}

El objetivo general de esta investigación es comparar los niveles de rendimiento entre los estudiantes repetidores y los no repetidores.

De este objetivo general se desprenden los siguientes objetivos específicos:

- $\quad$ Realizar un análisis descriptivo de la muestra.

- $\quad$ Analizar las puntuaciones en el rendimiento de la muestra.

- Comparar las puntuaciones en el rendimiento obtenidas por los escolares no repetidores y por los escolares repetidores.

\section{MATERIAL Y MÉTODOS Instrumento}

Para la investigación se utiliza la EGD realizada por el ministerio de educación en 2009. Esta tiene por objetivo contribuir a la mejora de la calidad de la educación mediante la evaluación de los aprendizajes de los estudiantes y de los procesos de mejora e innovación educativa. Para ello, concretamente, la EGD realizada en 2009 evalúa mediante exámenes ordinales el nivel de adquisición de las competencias básicas en Lingüística, Matemáticas, conocimiento e Interacción con el mundo físico y, Social-ciudadana (rendimiento académico). La prueba fue realizada a nivel nacional, contestada en cualquier idioma oficial (castellano, catalán, gallego, valenciano 0 vasco) y en el nivel de cuarto de Educación Primaria. La EGD recoge información relativa a los escolares, centros, profesores y padres ${ }^{0}$ Para esta investigación se utiliza la información de Idioma, sexo, repetición y las puntuaciones obtenidas en cada una de las pruebas de rendimiento académico.

\section{Participantes}

La EGD fue contestada por 28798 escolares de los cuales se eliminaron en primer lugar a los que no reportaban sexo $(n=27466)$. Después, se eliminaron los que no reportaban edad $(n=$ 26295). A continuación, se eliminaron los que no reportaban alguna de las variables de interés $(n=25295)$. Finalmente, se eliminaron los escolares nacidos en el 2000 (matrícula adelantada), quedando así una muestra de 25245. 


\section{Métodos}

Para verificar la existencia de diferencias estadísticamente significativas, entre los escolares repetidores y no repetidores, se utilizará la prueba de diferencia de medias de Welch, que compara las medias de dos muestras, como alternativa a prueba t de Student. Se usa especialmente con muestras con $n$ diferentes. Parte de la hipótesis nula $\left(H_{0}\right)$ de que las medias de los grupos de la muestra son iguales y la hipótesis alternativa $\left(H_{1}\right)$ es que las medias de los grupos de la muestra son diferentes.

Para verificar el tamaño del efecto se utiliza la d de Cohen. El tamaño del efecto puede ser entendido como "el grado en que el fenómeno está presente en la población" este puede ser considerado pequeño $(d>.20)$, mediano $(d>.50)$ y grande $(d>.80)$ (Cohen, 1988).

Finalmente, la visualización gráfica de los datos se realizará mediante la metodología HJ-Biplot. Los métodos biplot son una representación gráfica en bajo dimensión de una matriz de datos multivariantes (Gabriel, 1971). Existen tres especificaciones de esta metodología, concretamente en esta investigación se utilizarla el HJ-Biplot, el cual representa con la misma calidad tanto las variables como los individuos (Galindo, 1986). Para su interpretación se debe tener en cuenta, por un lado, en el ángulo que hay entre variables (vectores), los cuales nos informan sobre la relación entre las variables, ángulos menores a 90 grados muestran correlaciones positivas, ángulos de 90 grados muestran independencia y ángulos superiores a 90 grados correlaciones negativas. Por otro lado, en las proyecciones de los puntos (individuos) sobre los vectores, los individuos más alejados del centro en sentido al vector poseerán puntuaciones más altas que los más alejados en dirección contraria (Urruticoechea \& Vernazza, 2019).

\section{RESULTADOS}

\section{Características de la muestra}

En este apartado se muestran los principales resultados descriptivos univariantes y bivariantes de la investigación.

Variables sociodemográficas: de los 25245 escolares que componen la muestra, se tiene que el $49.16 \%$ son de sexo femenino y el $50.84 \%$ masculino.

Tabla 1. Composición de la muestra (sexo, lengua y repetición)

\begin{tabular}{lcccc}
\hline & \multicolumn{2}{c}{ Niñas } & \multicolumn{2}{c}{ Niños } \\
& No repetidores & Repetidores & No repetidores & Repetidores \\
\hline Castellano & 8964 & 585 & 9213 & 809 \\
Catalán & 1340 & 77 & 1275 & 109 \\
Gallego & 612 & 33 & 602 & 48 \\
Valenciano & 171 & 1 & 178 & 10 \\
Vasco & 612 & 15 & 553 & 38 \\
\hline
\end{tabular}

Fuente: Elaboración propia

Como se observa en la Tabla 1, el $93.2 \%$ de la muestra es no repetidora y el $6.8 \%$ es repetidora, cabe resaltar que entre los escolares repetidores el mayor porcentaje lo obtienen los de sexo masculino con el $58.8 \%$. También se puede ver como la distribución por lengua es: el $77.5 \%$ para castellano, el $11.1 \%$ para catalán, el $5.1 \%$ para gallego, el $1.4 \%$ para valenciano y el $4.8 \%$ para vasco. 
Competencias: Comparando las competencias por grupos (Tabla 2) se puede observar como los no repetidores obtienen, en media, mayores puntuaciones que los escolares repetidores.

En los dos casos y para todos los componentes se verifican distribuciones normales $(-1<$ $S k<1)$ y $(-1<k u<1)$. Se destaca que el rango de puntuaciones es mayor para los escolares no repetidores, los cuales tienen mínimos más bajos y máximos más altos, para todas las competencias, que los escolares repetidores.

Tabla 2. Descriptivos univariantes - Competencias (No repetidores/ repetidores)

\begin{tabular}{|c|c|c|c|c|c|c|c|}
\hline No repetidores & Mín & $Q_{1}$ & $Q_{2}$ & $\bar{x}(d s)$ & $Q_{3}$ & Máx & $S k(k u)$ \\
\hline 1. Lingüística & 162.70 & 448.05 & 511.95 & $512.22(90.86)$ & 576.55 & 822.92 & $-.02(-.27)$ \\
\hline 2. Matemáticas & 191.50 & 446.06 & 503.20 & $511.16(90.36)$ & 572.26 & 838.28 & $.28(-.16)$ \\
\hline 3. Mundo físico & 154.48 & 450.07 & 513.92 & $511.87(89.70)$ & 577.11 & 815.21 & $-.17(-.18)$ \\
\hline $\begin{array}{l}\text { 4. Social y } \\
\text { ciudadana }\end{array}$ & 171.99 & 452.77 & 518.89 & 511.53 (89.99) & 575.85 & 763.48 & $-.34(-.22)$ \\
\hline Repetidores & Mín & $Q_{1}$ & $Q_{2}$ & $\bar{x}(d s)$ & $Q_{3}$ & Máx & $S k(k u)$ \\
\hline 1. Lingüística & 189.37 & 388.78 & 440.71 & $443.64(79.36)$ & 494.84 & 731.05 & $-.10(-.20)$ \\
\hline 2. Matemáticas & 200.95 & 406.06 & 449.50 & 452.94 (73.14) & 493.95 & 731.40 & $.35(.86)$ \\
\hline 3. Mundo físico & 192.11 & 391.19 & 445.11 & $445.68(82.58)$ & 498.37 & 704.78 & $-.03(-.02)$ \\
\hline $\begin{array}{l}\text { 4. Social y } \\
\text { ciudadana }\end{array}$ & 180.96 & 383.36 & 448.89 & $446.46(87.56)$ & 507.35 & 708 & $-.02(-.24)$ \\
\hline
\end{tabular}

Fuente: Elaboración propia

Para finalizar este apartado, en la Tabla 3 se presentan las correlaciones de Pearson, observando que todas las competencias correlacionan positivamente entre ellas y que esta correlación es fuerte. La relación más débil se da entre social y ciudadana y lingüística (.57) y la más fuerte entre Mundo físico y Lingüística (.68) y entre Mundo físico y Social y ciudadana (.68).

Tabla 3. Matriz de correlación - competencias

\begin{tabular}{lccccc}
\hline & 1 & 2 & 3 & 4 & 5 \\
\hline 1. Lingüística & - & & & & \\
2. Matemáticas & $.61^{*}$ & - & & & \\
3. Mundo físico & $.68^{*}$ & $.62^{*}$ & - & & \\
4. Social y ciudadana & $.66^{*}$ & $.57^{*}$ & $.68^{*}$ & - & \\
\hline
\end{tabular}

$* \mathrm{p}<.001$

Fuente: Elaboración propia

\section{Prueba de Welch}

Teniendo en cuenta los valores reportados en la Tabla $4(p<.001)$ se puede asegurar que existen diferencias de medias y que estas son a favor de los escolares no repetidores $(t>0)$ en todas las competencias. Finalmente, los tamaños del efecto obtenidos muestran que estos son medio/altos para todas las competencias $(.70<$ Cohen'sd $<.80)$. 
Tabla 4. Welch's t test para datos independientes

\begin{tabular}{lcccc}
\hline & $t$ & $d f$ & $p$ & Cohen $^{\prime}$ sd \\
\hline 1. Lingüística & 34.28 & 2070.03 & $<.001$ & .80 \\
2. Matemáticas & 31.35 & 2129.65 & $<.001$ & .71 \\
3. Mundo físico & 31.94 & 2034.13 & $<.001$ & .77 \\
4. Social y ciudadana & 29.73 & 2000.61 & $<.001$ & .73 \\
\hline
\end{tabular}

Fuente: Elaboración propia

\section{HJ-Biplot}

Por último, se presentan los resultados obtenidos al analizar las competencias medidas en la EGD mediante la metodología HJ-Biplot.

En las figuras 1 y 2 se muestra como la variabilidad explicada (considerando 2 ejes) es del $80 \%$ $(E j e 1+E j e 2)$. También se observa que mediante estos dos ejes se pueden explicar las relaciones multivariantes existentes entre todas las competencias del estudio.

Figura 1. Hj-Biplot (competencias y escolares)

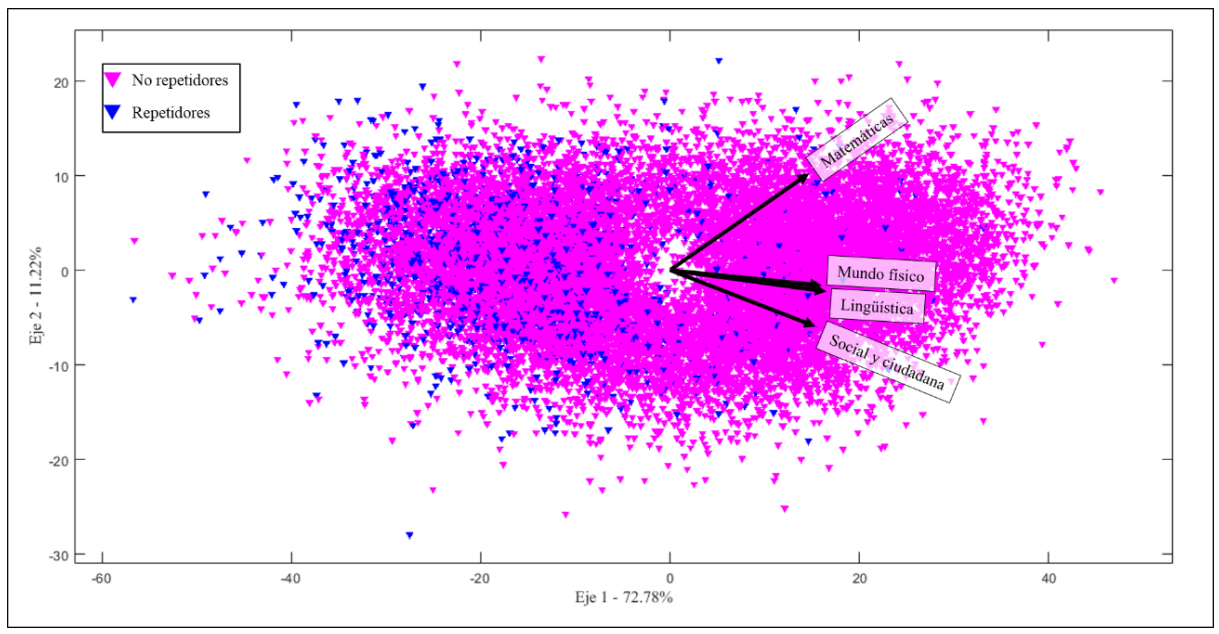

Concretamente, en la Figura 1 se puede observar, por un lado, que los escolares repetidores y no repetidores se encuentran mezclados por toda la superficie, pero la mayor concentración de los repetidores se da en las puntuaciones más bajas de la figura. Por otro lado, se distingue que en las puntuaciones más altas no hay presencia de repetidores. 


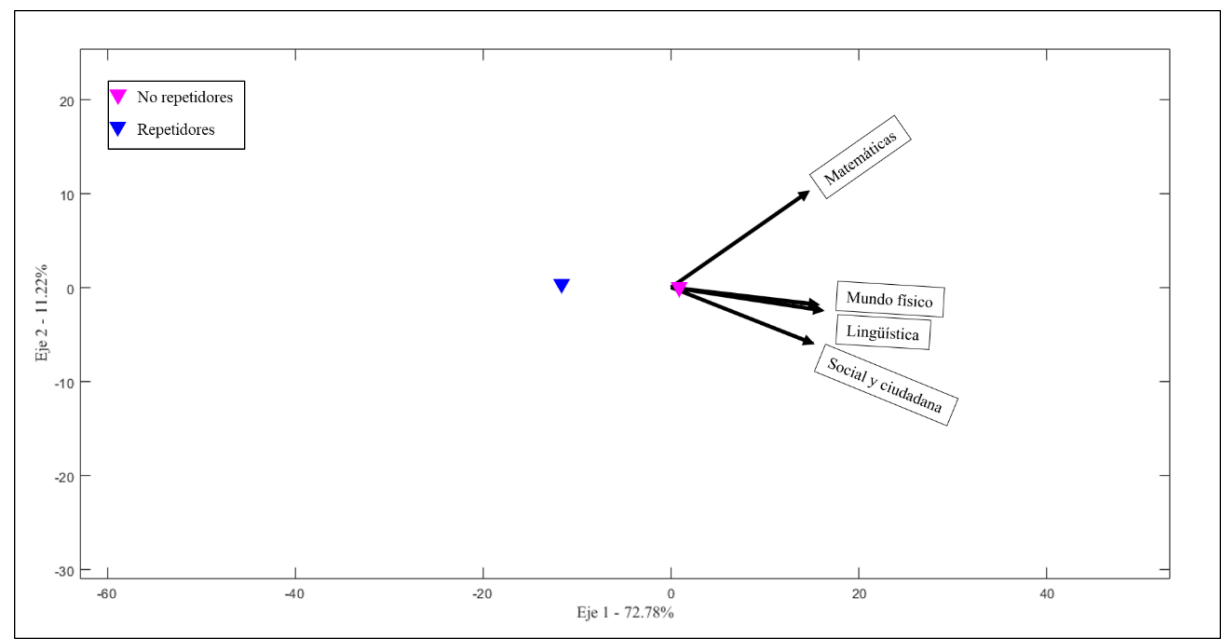

Observando los centroides, (punto medio de las distancias de todos los puntos de cada grupo) se distingue que las puntuaciones medias de los escolares no repetidores son más altas que la de los escolares repetidores.

Finalmente, en cuanto a la relación multivariante de las competencias estudiadas (vectores) en las dos figuras se destaca que todas presentan correlaciones positivas, la más fuerte se da entre mundo físico y Lingüística y la más débil entre Matemáticas y Social y ciudadana.

\section{CONCLUSIONES}

De los resultados obtenidos en este trabajo es posible concluir que la repetición, en la mayoría de los casos, no sirve para equiparar el rendimiento al de los escolares no repetidores, ya que, de media obtienen puntuaciones más bajas en todas las competencias que los escolares que no han repetido ningún año. Por lo que con esta muestra es posible asegurar que la repetición no cumple con el objetivo que tiene marcado de nivelación.

\section{DISCUSIÓN}

Una vez confirmado que la repetición no es un mecanismo adecuado de nivelación, entendiendo que la repetición en España se ha convertido en la característica más evidente de la injusticia educativa (Gortazar, 2019) y que, por lo tanto, se atenta directamente con el objetivo de desarrollo sostenible, propuesto por la Organización de las Naciones Unidas para la Educación, la Ciencia y la Cultura (UNESCO), de "garantizar una educación inclusiva, equitativa y de calidad y promover las oportunidades de aprendizaje durante toda la vida para todos", se entiende necesario un cambio en las políticas, reforzando el cambio de metodologías que permita lograr la igualdad. Entre otras acciones, se podría proponer:

- Adaptar el sistema educativo a los sujetos y no que los sujetos tengan que adaptarse al sistema educativo (Cordero, 1985). 
- Flexibilizar los exámenes, evaluando a los escolares de una clase en diferentes momentos del año (Crawford et al., 2010).

- Implementar estrategias educativas para no disminuir la autoestima de los estudiantes con rendimiento académico bajo (Ando et al., 2019)

Como estas, hay otras acciones que se podrían llevar a cabo, para utilizar otros mecanismos de nivelación de las capacidades de los escolares con el nivel educativo al que asisten, evitando usar la repetición como estrategia. Para ello es necesario realizar investigaciones más profundas sobre las repercusiones de las diferentes acciones.

\section{Pasos a futuro}

Como pasos a futuro y con la intención de confirmar los resultados de esta investigación se propone:

- $\quad$ Analizar, mediante pruebas estandarizadas PISA actuales, las diferencias en el rendimiento de escolares repetidores y no repetidores.

- Realizar una caracterización socioeconómica del rendimiento en España atendiendo especialmente la variable de repetición.

- $\quad$ Analizar qué factores se asocian con las puntuaciones bajas en el rendimiento, haciendo hincapié en los repetidores.

- $\quad$ Observar las diferencias en el rendimiento de los escolares de países que no permiten la repetición y España.

\section{REFERENCIAS BIBLIOGRÁFICAS}

Ando, S., Usami, S., Matsubayashi, T., Ueda, M., Koike, S., Yamasaki, S., Fujikawa, S., Sasaki, T., Hiraiwa-Hasegawa, M., Patton, G., Kasai, K., \& Nishida, A. (2019). Age relative to school class peers and emotional well-being in 10-year-olds. PLOS ONE, 14(3), e0214359. https://doi.org/10.1371/journal.pone.0214359

Barbosa, Regina H. (1975). "El rendimiento y sus causas", en: Illich, et al. Crisis en la didáctica, Axis, Argentina.

Cabrera, L. (2019). Políticas educativas preventivas de la repetición de curso en la enseñanza obligatoria en España. Multidisciplinary Journal of Educational Research, 9(3), 227. https://doi.org/10.17583/remie.2019.4523

Cohen, J. (1988). Statistical power analysis for the behavioral sciences (2nd ed). L. Erlbaum Associates.

Cordero, J. A. (1985). Relación de la variable edad con las deficiencias de escolarización en la EGB. Infancia y Aprendizaje, 8(29), 95-104. https://doi.org/10.1080/02103702.1985.10822062

Crawford, C., Dearden, L., \& Meghir, C. (2010). When you are born matters: The impact of date of birth on educational outcomes in England (Working Paper Series) [Working Paper Series]. https://doi.org/10.1920/wp.ifs.2010.1006

Gabriel, K. R. (1971). The biplot graphic display of matrices with application to principal component analysis. Biometrika, 58(3), 453-467.

Galindo, P. (1986). Una alternativa de representación simultánea: HJ-Biplot. Questió, 10(1), 13-23.

González-Betancor, S. M., \& López-Puig, A. J. (2016). Grade Retention in Primary Education Is Associated with Quarter of Birth and Socioeconomic Status. PLOS ONE, 11(11), e0166431. https://doi.org/10.1371/journal.pone.0166431 
Gortazar, L. (2019). ¿Favorece el sistema educativo español la igualdad de oportunidades? ICE, Revista de Economía, 910. https://doi.org/10.32796/ice.2019.910.6917

Hemelt, S. W., \& Rosen, R. B. (2016). School Entry, Compulsory Schooling, and Human Capital Accumulation: Evidence from Michigan. The B.E. Journal of Economic Analysis \& Policy, 16(4). https://doi.org/10.1515/bejeap-2015-0219

Hughes, J. N., Cao, Q., West, S. G., Allee Smith, P., \& Cerda, C. (2017). Effect of retention in elementary grades on dropping out of school early. Journal of School Psychology, 65, 11-27. https://doi.org/10.1016/j.jsp.2017.06.003

Instituto de Evaluación (España). (2010). Evaluación general de diagnóstico 2009. Instituto de Evaluación.

Ley Orgánica 2/2006, de 3 de mayo, de Educación. (s. f.). 110.

Magnuson, K. A., Meyers, M. K., Ruhm, C. J., \& Waldfogel, J. (2004). Inequality in Preschool Education and School Readiness. American Educational Research Journal, 41(1), 115-157. https://doi.org/10.3102/00028312041001115

Mantzicopoulos, P., \& Morrison, D. (1992). Kindergarten Retention: Academic and Behavioral Outcomes Through the End of Second Grade. 29(1), 182-189.

Peña, P. A. (2017). Creating winners and losers: Date of birth, relative age in school, and outcomes in childhood and adulthood. Economics of Education Review, 56, 152-176. https://doi.org/10.1016/j.econedurev.2016.12.001

Roldán Prego, L., Rodríguez Llorente, C., \& Vieites Lestón, T. (2019). El papel de la repetición y el rendimiento académico en el abandono escolar temprano. International Journal of Developmental and Educational Psychology. Revista INFAD de Psicología., 1(2), 83-92. https://doi.org/10.17060/ijodaep.2019.n2.v1.1674

Stearns, E., Moller, S., Blau, J., \& Potochnick, S. (2007). Staying Back and Dropping out: The Relationship between Grade Retention and School Dropout. Sociology of Education, 80(3), 210240. JSTOR.

Urruticoechea, A., \& Vernazza, E. (2019). Relación entre la educación y el trabajo infantil en niños y adolescentes de Uruguay. International Journal of Developmental and Educational Psychology. Revista INFAD de Psicología., 4(1), 451. https://doi.org/10.17060/ijodaep.2019.n1.v4.1611

Ventura-León, J. (2018). Otras formas de entender la d de Cohen. Revista Evaluar, 18(3). https://doi.org/10.35670/1667-4545.v18.n3.22305 
